\title{
Universitarias argentinas. Desafíos para contarlas
}

Gómez Molla, Rosario

Universidad Nacional de La Plata-Instituto de Investigaciones en Humanidades y Ciencias Sociales (IDIHCS)-CONICET, Argentina rosariogomezmolla@gmail.com

Cita sugerida: Gómez Molla, R.(2018). Universitarias argentinas. Desafíos para contarlas Anuario del Instituto de Historia Argentina, 18 (1), e064. https://doi.org/10.24215/2314-257Xe064

Recibido: 23 Febrero 2018 - Aceptado: 20 Mayo 2018 - Publicado: 28 de junio de 2018

(c) (1) (5) Esta obra está bajo licencia Creative Commons Atribución-NoComercial-CompartirIgual 4.0 Internacional cc) $\mathrm{http}: / /$ creativecommons.org/licenses/by-nc-sa/4.0/deed.es_AR 


\section{Universitarias argentinas. Desafíos para contarlas}

Argentine Universities Statistics. Women matters

Rosario Gómez Molla

Universidad Nacional de La Plata-Instituto de

Investigaciones en Humanidades y Ciencias Sociales

(IDIHCS)-CONICET, Argentina

rosariogomezmolla@gmail.com

\section{ReSUMEN:}

Los trabajos que analizan, desde una perspectiva histórica, la participación de las mujeres en la universidad recurren habitualmente a una fuente estadística de gran valor: Evolución de la mujer en las profesiones liberales en Argentina (1900-1965), lanzada en 1965 por la Oficina Nacional de la Mujer. En primer lugar, nos interesa examinar las diversas fuentes estadísticas que se utilizan para analizar la población universitaria a lo largo del siglo XX. Luego, revisaremos los datos que la publicación de la Oficina Nacional de la Mujer recoge y señalaremos sus aportes y limitaciones. Por último, presentaremos el trabajo de reconstrucción de la información relativa a la población femenina de la Universidad Nacional de La Plata que hemos realizado, para el período 1905-1972.

Palabras Clave: Estadísticas, Mujeres universitarias, Argentina.

\section{Abstract:}

The studies that analyse women's participation at universities from a historical perspective often utilize a highly valuable statistical source: Evolución de la mujeren las profesionesliberalesen Argentina (1900-1965), published in 1965 by the Women's National Council. Firstly we are interested in examining the diverse statistical sources that are used for analyzing university population throughout the 20th century. Secondly we will revise the data which the Women's National Council's publication operates, and we will clarify its contributions and limitations. Finally, we will introduce the reconstruction study we have elaborated with the information relative to the female population at the University of La Plata concerning the period between 1905 and 1972.

KEYWORDS: Statistics, Female university students, Argentina.

\section{INTRODUCCIÓN}

Desde los inicios del Estado argentino, la formación impartida por las universidades, primero la de Córdoba y luego la de Buenos Aires, se caracterizó por sus contenidos orientados a la actividad política y la administración estatal. La voluntad de las clases dirigentes asignaba a la educación universitaria un rol central en la formación de futuros cuadros de gobierno, situación que hacia fines del siglo XIX comenzaba a provocar ciertas tensiones. El perfil de las carreras -según señala Buchbinder (2010) - era objeto de controversia: por un lado, estaba la postura de las elites y, por el otro, la demanda de una sociedad que entendía a la universidad como el medio para obtener un título profesional, y así perspectivas de mejores empleos. El binomio educación universitaria/ascenso social comenzaba a consolidarse como horizonte entre los sectores medios urbanos, situación que se veía reflejada en una matrícula y egresos cada vez más voluminosos (Cano, 1985; Oficina Nacional de la Mujer, 1970).

Si bien la Reforma Universitaria de 1918 tuvo un impacto considerable en la ampliación de las posibilidades de acceso a la universidad, el crecimiento "explosivo" ocurrió durante la gestión peronista, "mientras en 1945 había tres estudiantes universitarios cada mil habitantes, en 1955 llegaban aproximadamente a ocho" (Buchbinder, 2010, p. 159). Es decir que la cantidad de alumnos había crecido casi tres veces en diez años. Movimientos similares se constatan en los egresos de las universidades nacionales: en 
1940 se otorgaron 2830 diplomas, quince años después, en 1955, esa cifra había ascendido a 5820, y alcanzó, en 1965, los 10097 egresos (Oficina Nacional de la Mujer, 1970).

Entre los elementos que motivaron este proceso Buchbinder (2010) señala: la suspensión del cobro de los aranceles universitarios en 1949, la puesta en marcha de un sistema de becas dirigidas a estudiantes de escasos recursos, la supresión del examen de ingreso y la expansión de la matrícula secundaria. Este último es estudiado por Cammarota (2014), quien indica que la "democratización" del ciclo secundario se debió, en primer lugar, a la "centralización del sistema educativo", mediante la creación del Ministerio de Educación de la Nación. Luego, se puso en marcha un conjunto de medidas que impulsaron aquel fenómeno, entre las que se cuentan: la creación de opciones de albergue para jóvenes, el otorgamiento de becas, y la ampliación de la oferta educativa en su variante técnica especialmente. Además, el crecimiento acelerado de la educación universitaria no era una característica exclusiva de Argentina: la tendencia internacional iba en la misma dirección a medida que se consolidaba, en los años sesenta, un discurso que caracterizaba a la "educación como factor de desarrollo" (Perez Lindo, 1985).

Sabemos que las mujeres formaron parte de este proceso, aunque resulta dificultoso caracterizar algunos aspectos de su participación. Los datos relativos a la matrícula universitaria no han incluido la distinción entre varones y mujeres sino hasta los años cuarenta; $y$, aun a partir de allí, la información con la que contamos es fragmentaria y disímil. Así, los intentos por reconstruir la participación femenina en el largo plazo, desde sus comienzos hasta la actualidad, se encuentran con grandes obstáculos.

En este contexto, un estudio estadístico elaborado por la Oficina Nacional de la Mujer en los años sesenta se presenta como una fuente de gran valor para quienes nos proponemos aquella tarea. Publicado por primera vez en 1965 y reeditado en 1970, Evolución de la mujer en las profesiones liberales en Argentina, años 1900-65, recoge datos sobre la totalidad de los títulos otorgados por las universidades nacionales de nuestro país en el período 1900-1965. Entre sus principales atributos se cuentan el largo de la serie, y la incorporación de la distinción por género y por rama de estudio.

Su análisis revela la importante participación de las mujeres en el crecimiento acelerado de la población universitaria en los años cincuenta y sesenta. Además, habilita la distinción por género de las orientaciones vocacionales: vemos, por ejemplo, que la preponderancia de las carreras de Medicina y Derecho, que se constata a lo largo de todo el período cuando observamos la titulación total, no es tal si tomamos únicamente la titulación femenina. Si bien Medicina sí estuvo en el podio durante toda la serie, el caso de Derecho muestra un giro notable en la orientación de las universitarias en los últimos años de la década de 1950, al posicionarse en segundo lugar en el orden de la titulación femenina, mientras que en las décadas previas había estado muy lejos de las tres carreras con más egresos: Medicina, Filosofía, y Farmacia y Bioquímica.

Estos movimientos contribuyeron en la configuración de nuestro objeto de estudio; a saber, la profesionalización femenina en el campo del Derecho en los años sesenta. Si bien no todos los trabajos sobre procesos de profesionalización deben abordar temáticas vinculadas a la universidad, puesto que no todas las profesiones necesitan de la acreditación universitaria, en nuestro caso nos parecía importante comenzar por allí, dado que consiste en el primer paso de las profesiones jurídicas. Además, este aspecto del proceso de profesionalización nos ayudó a definir nuestro período de estudio. Cuando constatamos el crecimiento destacado de la titulación femenina en Derecho entre fines de los años cincuenta y principios de los sesenta, decidimos focalizar nuestra pesquisa en ese contexto de expansión de la presencia de mujeres en el mundo jurídico.

Las líneas que siguen se enmarcan en el desarrollo de aquella investigación doctoral. Aquí, nos interesa dar cuenta de los obstáculos con los que nos encontramos al intentar reconstruir algunos aspectos del paso por la universidad de las abogadas, escribanas y procuradoras, sobre las que nos proponemos indagar. Estas dificultades nos han permitido dimensionar los desafíos de abordar desde un enfoque cuantitativo e histórico a la población universitaria femenina en general, como lo desarrollaremos a continuación. 
En el primer apartado analizaremos algunas de las fuentes estadísticas sobre población universitaria. Si bien no contamos con las herramientas para presentarlas como las únicas disponibles, sí podemos afirmar que son las fuentes citadas por los textos más difundidos sobre la temática. Luego, examinaremos los datos que presenta la publicación de la Oficina Nacional de la Mujer, y señalaremos sus aportes y limitaciones. Por último, presentaremos el trabajo de reconstrucción de las estadísticas de la Universidad Nacional de la Plata que hemos realizado.

\section{CONTAR A LAS UNIVERSITARIAS}

\section{Las fuentes estadísticas}

Como mencionamos en la introducción, la gran expansión de la población universitaria en los años cincuenta y sesenta no era un fenómeno exclusivo de Argentina. A nivel internacional ocurrían movimientos similares, en tanto se vinculaba a la educación superior con objetivos que la trascendían. Chiroleu (2006) analiza la recepción que tuvo en nuestro país la denominada "teoría del capital humano", que incorporaba en el cálculo de la productividad económica a la educación como un elemento central. En este marco, diversas acciones, fomentadas en gran medida por organismos internacionales, impulsaron la idea de "planificación educativa", que tuvo como correlato institucional la creación de áreas de gobierno destinadas a dicho fin.

Las universidades, por supuesto, también fueron parte de la reflexión en torno al sistema educativo, como lo demostró el crecimiento de las carreras de Educación, en los años cincuenta y sesenta, según señala Suasnábar (2014). El autor se ocupa de analizar los debates que tenían lugar en el período en aquel campo disciplinar haciendo foco en la recepción que tuvieron algunas "ideas del exterior", entre las cuales cuenta a la educación comparada y sus planteos en torno a la importancia de la recopilación de información de los sistemas educativos, tarea que asimismo impulsaban organismos internacionales como la UNESCO (Suasnábar, 2014).

Estos movimientos ocurrieron a la par de un proceso de institucionalización de la producción estadística que tomó vigor con el proyecto desarrollista de los años sesenta y se consolidó con la creación, en 1968, del Instituto Nacional de Estadísticas y Censos (INDEC), bajo la órbita del Consejo Nacional de Desarrollo (CONADE) (Daniel, 2009, pp. 172-173). Ese mismo año, el CONADE, instituido en 1961 como organismo de Presidencia de la Nación, publicó un informe detallado sobre el sistema educativo argentino, en el cual la información estadística ocupó un lugar central. Educación, recursos humanos y desarrollo económicosocial se propuso como un diagnóstico útil para la planificación educativa en aras del "desarrollo", y cabe señalar que fue elaborado en conjunto con "expertos" de la Organización para la Cooperación y Desarrollo Económicos (OCDE) (CONADE, 1968, p. VIII).

Este informe no fue el único trabajo que buscó por aquellos años caracterizar, a partir de información cuantitativa, diversos aspectos del sistema universitario. Dentro de los reportes que publicó el Departamento de Estadísticas del Ministerio de Educación de la Nación, en los años sesenta, existieron apartados dedicados a la educación superior. Además, Santos Sharpe (2016), en un trabajo en el que historiza el abordaje sobre el abandono universitario en Argentina, señala que las primeras investigaciones al respecto vieron la luz en los años sesenta. Entre ellas el autor cuenta los escritos de Aráoz (1968), Graciarena (1961) y Germani y Sautu (1965) que, si bien se inscribían en corrientes de pensamiento distintas - como señala el autor -, compartían el valor que le asignaban a determinados datos estadísticos a la hora de analizar el fenómeno universitario.

El informe del CONADE se preocupó por el presente del sistema educativo, y por ello recogió datos sobre un período acotado, 1952-1965, los cuales no permiten visualizar movimientos en el largo plazo. Distinto fue el caso de La educación superior en Argentina (Cano, 1985). Editada en 1985 por la UNESCO, esta obra se interesó por el desarrollo histórico del nivel universitario en nuestro país, con un anexo estadístico con 
datos desde 1910. Sin embargo, ambas publicaciones sí coincidieron en sus críticas a las insuficientes fuentes de información educativa. El informe del CONADE se refiere a la matrícula universitaria y señala, por un lado, la escasez de datos y, por el otro, la diversidad de criterios en la definición de alumno regular, central a la hora de calcular la matrícula (CONADE, 1968, p. 112). El trabajo de Cano, por su parte, le achaca las falencias a la heterogeneidad del sistema superior, que ve reflejada en la disponibilidad de las fuentes, en lo que denomina como "atomización" de las mismas (Cano, 1985).

Entre las principales fuentes que utilizaron ambos informes se cuentan las publicaciones del Departamento de Estadística del Ministerio de Educación de la Nación y las del Consejo de Rectores de las Universidades Nacionales. Resulta extraño que, ni los escritos de Cano en la década de los ochenta, ni el informe del CONADE a fines de los sesenta hayan citado a la publicación de la Oficina Nacional de la Mujer, que se editó por primera vez en 1965, como mencionamos más arriba. Si bien desconocemos los motivos de esta omisión, no podemos dejar de señalarla, ya que Evolución de la mujer en las profesiones liberales hubiese sido una fuente muy valiosa para estos informes, entre cuyos objetivos estaba la caracterización de la población universitaria.

\section{Los datos que presentan}

El informe del CONADE se divide en tres partes, la primera dedicada al "diagnóstico de la situación educativa”. El capítulo II se ocupa de la "población escolar”, presentando las tasas de escolarización en los distintos niveles: pre-primaria, primaria, media y superior-universitaria. Las tasas fueron construidas a partir de las matrículas por nivel educativo sobre la población total en "edad normal" correspondiente a cada nivel. Afortunadamente, se incluyó en el análisis la variable de género. Sabemos así que, en 1952, la tasa de escolarización femenina en el nivel superior-universitario era igual a 2,8 y que, en 1965, había trepado a 5,7. Al comparar estas cifras con las correspondientes a los varones, vemos lo relevante del incremento de la participación de las mujeres en la educación superior: entre 1952 y 1965 la tasa de escolarización masculina sólo aumentó 0,1, cuando pasó de 7,7 a 7,8 (CONADE, 1968, p. 80).

La distribución de la población de las universidades nacionales por rama de estudio es un aspecto que el informe desarrolla con especial interés. La comparación con países europeos está a la orden del día cuando se analiza la relativamente baja participación de las "ramas científico-técnicas" en Argentina. En este sentido, el informe señala con preocupación la notable preponderancia de Medicina y Derecho, en el período analizado (1952-1965).

En cuanto a la participación de las mujeres en la distribución por ramas de estudio, el informe sólo presenta datos de la matrícula de 1963. Allí, se clasifican las disciplinas en tres grandes grupos - cientificas y tecnológicas, ciencias médicas y ciencias sociales y humanidades -, en los que la población femenina representó el 15,9\%, el $31,5 \%$ y 40,7 \% del total del alumnado de cada grupo, respectivamente. De este modo, en 1963 las ciencias sociales se posicionaban como el ámbito universitario con mayor cantidad de estudiantes mujeres y, a su vez, como la principal opción de las mujeres, al representar el 61,6 \% del total de la población universitaria femenina (CONADE, 1968, p. 140).

Como vemos, el informe del CONADE arroja algunos datos discriminados por género, como también lo hace el escrito editado por la UNESCO. En un apartado dedicado a la feminización de la educación superior, Cano (1985) destaca el aumento notable de la participación femenina sobre el total de la población escolar, la cual llega incluso a superar a la masculina en 1983, cuando alcanza el $54 \%$. En cuanto a la educación superior, el autor hace hincapié en la heterogeneidad de la presencia de mujeres según se trate de educación superior universitaria o no universitaria y, dentro de cada variante, según las carreras. Al analizar los datos del Censo Nacional de Población y Vivienda de 1980, Cano advirtió que las carreras no universitarias vinculadas a la docencia, cuya duración variaba entre los dos y los cuatro años, tenían una matrícula "abrumadoramente femenina" (Cano, 1985, p. 45). 
El anexo estadístico ofrece más información sobre la población universitaria, aunque la variable de género aparece sólo en algunos cuadros y, lamentablemente, en períodos cortos, o años puntuales, y en términos porcentuales. En relación a la matrícula, cuenta con información de los años 1963 y 1983 relativa a la participación de las mujeres en los subsistemas de la educación superior, universitario y no universitario; así pues, sabemos que, mientras en 1963 las mujeres representaban el $32 \%$ de la matrícula universitaria, veinte años después esa cifra había ascendido al $44 \%$ (Cano, 1985, p. 117).

El anexo también ofrece datos de la matrícula por ramas de estudio en los años 1960 y 1980 . Aquí es interesante observar la merma de alumnos, varones y mujeres, en la rama de las ciencias médicas: en 1960, un 29,7 \% de los varones matriculados en la universidad y un 31,4\% de las mujeres optaban por carreras relacionadas a las ciencias médicas. Veinte años después, en 1980, el porcentaje de varones había descendido a $11,1 \%$ y el de las mujeres a $17 \%$ (Cano, 1985, p. 129). En otro cuadro se suma la variable establecimiento público o privado para 1980: vemos entonces que el porcentaje de mujeres en relación al total de la matrícula en las universidades privadas era, en ese año, levemente mayor al de las universidades públicas, con un $46 \%$ y un $44 \%$ respectivamente. Si observamos esa relación por orientación de los estudios, es llamativo lo que ocurrió en la rama de las ciencias médicas, con un $70 \%$ de mujeres en las universidades privadas, frente a un $53 \%$ en las públicas (Cano, 1985, p. 129). Sería interesante indagar sobre los motivos de esta diferencia y preguntarse, por ejemplo, cómo funcionaron las dinámicas inclusivas y/o expulsivas de las mujeres en cada facultad - en particular - y en cada universidad - en general -, y cómo éstas se vincularon con la historia y tradición de las universidades públicas en nuestro país.

Si bien estos datos nos permiten observar algunos rasgos de la participación de las mujeres en los estudios universitarios, no son suficientes para caracterizar su evolución a través del tiempo, ya que no refieren a un período extenso. Existieron dos trabajos, publicados en los años ochenta, que sí se propusieron esta tarea: Evolución de la participación universitaria femenina en Argentina (1940-1980) (García Frinchaboy, 1981) y Situación educativa de las mujeres en Argentina (Maglie y García Frinchaboy, 1988). En el primero, García Frinchaboy dedicó buena parte a la reconstrucción de la matrícula femenina, entre 1941 y 1978. Para ello, recolectó datos de fuentes del Ministerio de Educación de la Nación, aunque con grandes dificultades debido a los vacíos estadísticos. Tal es así que en un período de treinta y siete años tuvo que estimar los números de nueve, entre 1949 y 1957. Aun con esta falencia, el trabajo de García Frinchaboy representa un aporte en tanto ofrece números absolutos de la matrícula femenina en la universidad por casi treinta años, de 1941 a 1948, y de 1958 a 1978.

Situación educativa de las mujeres en Argentina, escrito que tiene a García Frinchaboy como coautora, analizó la participación femenina en todos los niveles educativos. En el apartado dedicado a la educación universitaria se tomaron como fuentes el trabajo de García Frinchaboy y la publicación de la Oficina Nacional de la Mujer. El trabajo se interesó en dos dimensiones del fenómeno: por un lado, en la participación creciente de las mujeres en el nivel universitario a lo largo de los años, y, por el otro, en la distribución de la matrícula femenina por rama de estudio. Así, clasificó las orientaciones en categorías tales como "carreras típicamente femeninas", aquellas cuya matrícula está conformada en más de un $60 \%$ por mujeres, y "carreras típicamente masculinas", cuando más del $60 \%$ son varones. Al comparar la distribución de la matrícula en 1941 con la de 1978, las autoras constataron movimientos interesantes, entre los cuales se destacan la ampliación y la reorientación de las preferencias femeninas, que van ganando lugares en "carreras típicamente masculinas", como Derecho, por ejemplo (Maglie y García Frinchaboy, 1988, p. 33). El análisis de los egresos había arrojado conclusiones similares en Evolución de la mujer en las profesiones liberales, con el plus de analizar un período mucho más amplio, como veremos a continuación. 


\section{Sobre Evolución de la mujer en las profesiones liberales en Argentina}

\section{La Oficina Nacional de la Mujer}

En un trabajo que se propone recopilar el conjunto de políticas estatales que "explícita o implícitamente" involucraron a las mujeres, Novick (1993) desarrolla el derrotero institucional de la Oficina Nacional de la Mujer.

En 1955, el gobierno de facto de la autodenominada "Revolución Libertadora" crea la Dirección Nacional de la Mujer, bajo la órbita del Ministerio de Trabajo y Previsión. Dos años más tarde la dependencia es suprimida, para ser creada nuevamente en 1958, durante el gobierno de Frondizi. La pertenencia ministerial no era casual, ya que entre sus objetivos se contaba el de brindar asesoramiento en lo referido a la "mujer que trabaja" (Novick, 1993).

En 1964, se transforma en el Departamento de la Mujer y, con el cambio de nombre, aparecen nuevas responsabilidades, tales como "fomentar la difusión de temas relativos a la mujer que trabaja" (Novick, 1993). En este marco, la dependencia lanza una serie de publicaciones que abordaban problemáticas ligadas a la participación de las mujeres en el mercado laboral y al ejercicio de sus derechos. Una de ellas fue Evolución de la mujer en las profesiones liberales en Argentina, 1900-1965.

Esta publicación se nutrió de los datos de titulación de las universidades nacionales que existían por aquellos años, con la excepción de la Universidad Tecnológica Nacional. Relevaron entonces información de ocho universidades: las de Buenos Aires, Córdoba, La Plata, Tucumán, Cuyo, del Litoral, del Nordeste y del Sur. Los datos recolectados, como ya hemos señalado, se refieren a los títulos otorgados por las instituciones entre 1900 y 1965. Es posible que esta elección se haya debido a la disponibilidad de la información, en contraposición a las dificultades para el relevamiento de la matrícula, como indicaba el informe del CONADE, por ejemplo. Además, los diplomas llevan la firma de la autoridad máxima de las universidades, razón por la cual cabe suponer la existencia de un registro unificado, lo cual sortea el obstáculo de la heterogeneidad de fuentes planteado por Cano.

Sin embargo, al parecer en un principio la Oficina aspiraba a recolectar datos de inscripciones. En 1962, en una nota dirigida al presidente de la Universidad Nacional de La Plata, en la que se le solicitaban los datos necesarios para confeccionar el estudio, la directora de la dependencia demandó no sólo la información relativa a los egresos, sino también las inscripciones por año, género y carrera, entre 1957 y 1961 . Aunque desconocemos los motivos por los cuales finalmente no publicaron esa información, podemos intuir que tuvieron dificultades en el acceso a la misma.

Incluso la recolección de la información de los egresos no fue tarea sencilla. Entre las fuentes universitarias que cita el estudio figuran un conjunto de áreas de la Universidad Nacional de Córdoba, lo que sugiere que la Oficina tuvo que establecer contacto con cada una de las facultades que integraban la Universidad para poder acceder a la totalidad de la información. También sabemos que, en 1963, personal de la dependencia visitó la Universidad Nacional de La Plata para indicarle al área correspondiente el modo en que debían remitir los datos solicitados. Esto se suma a los vaivenes políticos de los sesenta, que repercutieron en la Oficina tanto a nivel orgánico (con cambios de nombre y funciones) como en lo que respecta a las sucesivas funcionarias a cargo.

A pesar de las contrariedades, y luego de al menos tres años de trabajo, el estudio fue publicado en 1965 y prologado por la jefa del entonces Departamento de la Mujer, Lucía Leban de Calot, quien una fervorosa defensa del trabajo remunerado de las mujeres y sus efectos en la sociedad. En este sentido, afirmó que "promover a la mujer, es promover a la familia, promover a la familia es promover a la comunidad" (Oficina Nacional de la Mujer, 1970, p. 9). Esta no era una idea aislada en los años sesenta: según señala Cosse (2010), el empleo femenino era percibido por algunos sectores como un elemento que contribuía en la realización de la mujer madre, lo que a su vez - alegaban - favorecía la crianza de los hijos. 
Reeditado en 1970, por la ahora denominada Oficina Nacional de la Mujer, el prólogo a la segunda edición del estudio hizo especial hincapié en la relación entre estudios universitarios y desarrollo laboral de las mujeres, al sostener que "la presencia de la mujer en el proceso socioeconómico es uno de los signos que caracterizan la época en la que vivimos", y afirmar que "las profesiones liberales han abierto a la mujer un amplio panorama y le ofrecen las armas más aptas para competir en igualdad de condiciones con el hombre" (Oficina Nacional de la Mujer, 1970, p. 7). En este sentido, pareciera ser que el objetivo del documento no era sólo informativo, sino que se orientaba a la promoción del desarrollo profesional femenino.

\section{Evolución de los egresos de mujeres y varones}

El vínculo entre los estudios universitarios y el ascenso social formaba parte de un sentido común que compartían los sectores medios urbanos. Si hacia fines del siglo XIX la educación primaria fue presentada como una necesidad política más que económica -la formación de ciudadanos- (Lionetti, 2005), el nivel universitario se erigía, a lo largo de la primera mitad del siglo XX, como un hito propulsor del desarrollo laboral de las personas. Esta percepción seguramente contribuyó a la expansión de la matrícula universitaria, junto con el crecimiento del ciclo secundario que señalamos en la introducción.

Los datos sobre la titulación también arrojan luz sobre estas cuestiones, con el complemento -central en nuestro trabajo- de la variable de género. Como podemos observar en la Figura 1, las curvas de la evolución en la titulación representan un incremento mayormente sostenido en el período, con altibajos que no modifican la tendencia creciente. En este proceso, la titulación de las mujeres fue aumentando, primero lentamente y, luego, de manera más proporcional a los egresos totales. En especial en el último tramo cuando, en 1965, la titulación femenina era ocho veces más grande de lo que había sido en 1940, mientras que la total se había poco más que triplicado. Aunque no alcanzaba niveles de paridad con la titulación masculina, la brecha entre ambas se reducía cada vez más rápido.

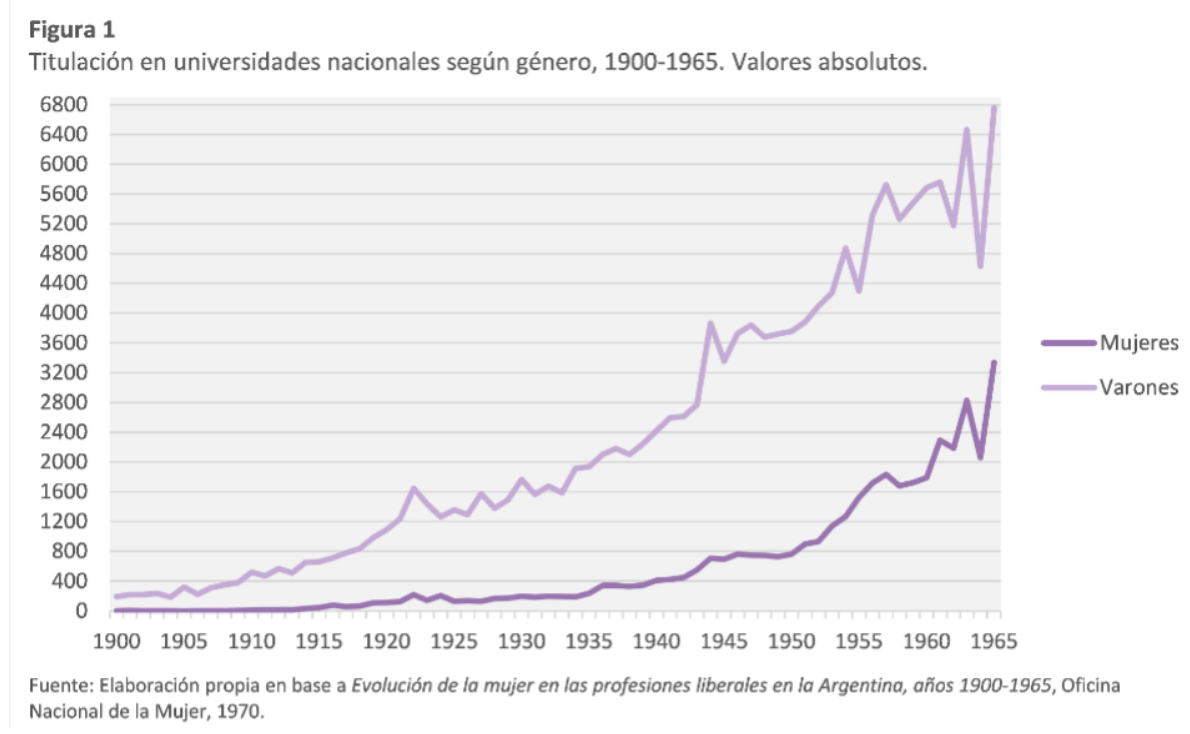

Las primeras graduadas debieron afrontar un contexto adverso para el trabajo de las mujeres fuera del hogar. En este sentido, si bien la clase dirigente consensuaba en los beneficios sociales que propiciaba la instrucción de las mujeres, por sus efectos sobre el bienestar de la familia la oposición al empleo femenino era férrea (Nari, 1995; Queirolo, 2010). Ya desde la escuela primaria, la formación impartida incluía en sus contenidos la distinción de roles por género. Guiaba a los varones hacia un futuro de participación en el 
mercado laboral y de ejercicio de sus derechos políticos, mientras que a las mujeres se las preparaba para el ámbito doméstico (Lionetti, 2005).

Así pues, no resulta extraño que las primeras egresadas fueran médicas, en general orientadas hacia especializaciones ligadas a la atención de mujeres y niños (Ramacciotti y Valobra, 2011) y profesoras (Lorenzo, 2016; Palermo, 2006). Ambas carreras se adecuaban en gran medida a lo que se esperaba de las mujeres por aquellos años: en el primer caso, a los discursos que las asociaban con el cuidado de los niños/as y la maternidad (Queirolo, 2008); en el segundo, se trataba de los efectos del proceso de feminización de la tarea docente, llevado a cabo a través de las escuelas normales (Barrancos, 2012). La primera egresada universitaria de nuestro país fue Cecilia Grierson, quien en 1889 recibió su diploma de médica. El contexto en que dio sus primeros pasos en el ámbito profesional no fue el más acogedor. Como señalan Ramacciotti y Valobra (2011), la oposición a las mujeres médicas reunía discursos "seudocientíficos" que buscaban "comprobar que las mujeres tenían una inferioridad biológica”. Incluso quienes se posicionaban a favor de las médicas apelaban a motivos que también retomaban una supuesta "naturaleza femenina", cuando destacaban su participación en las ramas de la pediatría y la ginecología. Por otro lado, en 1901, egresan cuatro mujeres de la Facultad de Filosofía y Letras de la Universidad de Buenos Aires. Lorenzo señala esta cuestión y analiza algunas de las tesis con las que finalizaron sus estudios, entre las cuales destaca a la presentada por Elvira López: El movimiento feminista.

Tiempo después, hacia mediados de siglo, algunas transformaciones tuvieron lugar. El aumento de la matrícula secundaria conllevó cambios en su composición: varió la opción de las mujeres por la escuela normal (Barrancos, 2008), y el nivel que más se expandió fue el bachillerato (Cammarota, 2014). La titulación universitaria reflejó cambios similares, tanto en términos cuantitativos, como a propósito de su composición.

La Figura 2 ilustra la evolución de los egresos de mujeres por grupo de carreras en los cuatro grupos con mayor titulación femenina durante el período en cuestión. A fin de normalizar los datos y poder elaborar totales nacionales, la Oficina optó por clasificar los títulos en diez grupos de carreras: Medicina, Farmacia y Bioquímica, Odontología, Derecho, Administración y Economía, Filosofía, Ciencias de la Educación y Profesorados, Matemáticas, Física, Química, Ciencias Naturales y Biológicas; Ingeniería, Arquitectura, y, por último, Agronomía y Veterinaria. En el gráfico mencionado figuran los cuatro grupos que más títulos les otorgaron a las mujeres a lo largo del período, a saber: Medicina, Farmacia y Bioquímica, Derecho, y Filosofía, Ciencias de la Educación y Profesorados.

Entre comienzos de siglo y 1957, las carreras con más egresos femeninos habían sido Medicina, con 5716 títulos otorgados; Filosofía, con 4452, y, en tercer lugar, Farmacia y Bioquímica, con 3608. Derecho, en cambio, con un total de 1891 títulos otorgados, quedaba relegada a un lejano cuarto puesto. Llamativamente, la tendencia se invirtió en 1958, cuando la titulación en Derecho despegó de manera acelerada, hasta posicionarse en el segundo puesto. Entre 1958 y 1965 Medicina otorgó 4915 títulos, seguida por Derecho, con 3582 egresos, luego Filosofía, con 2439, y, finalmente, Farmacia y Bioquímica con 2262 títulos otorgados.

Si comparamos la titulación femenina con la de los varones, ilustrada en la Figura 3, encontramos que, a diferencia de la primera, su composición se mantuvo constante a lo largo del período. Las cuatro carreras con más egresos masculinos fueron: primero Medicina, luego Derecho seguida por Ingeniería, y, por último, Administración y Economía. 


\section{Figura 2}

Titulación femenina en las cuatro orientaciones que otorgaron la mayor cantidad de títulos a mujeres, 1900-1965. Valores absolutos.

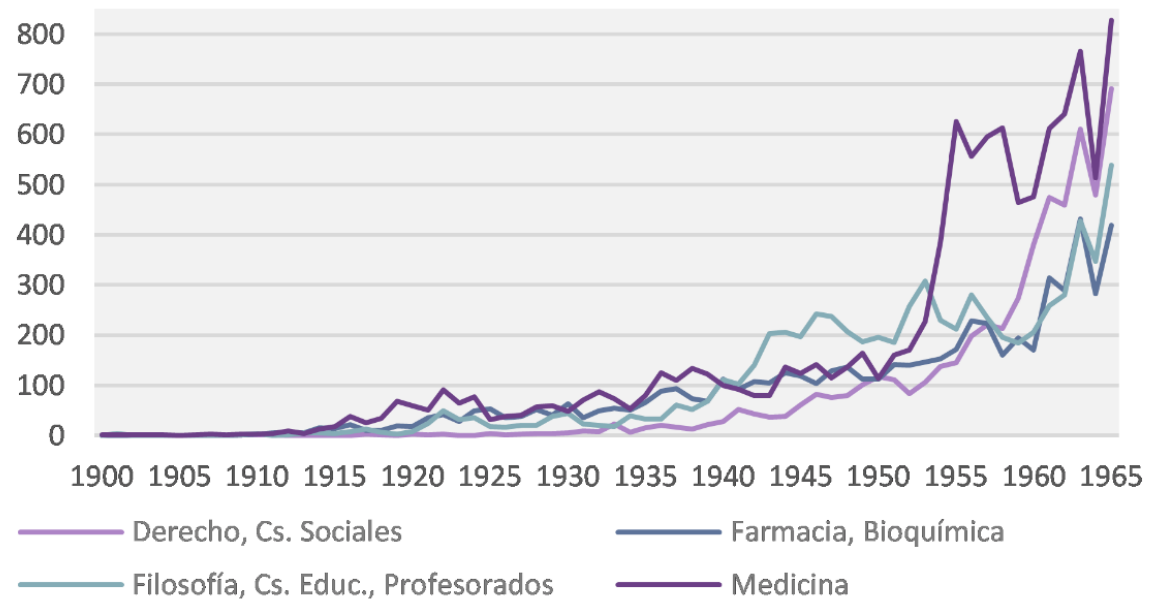

Fuente: Elaboración propia en base a Evolución de la mujer en las profesiones liberales en la Argentina, años 1900-1965, Oficina Nacional de la Mujer, 1970.

\section{Figura 3}

Titulación masculina en las cuatro orientaciones que otorgaron la mayor cantidad de títulos a varones, 1900-1965. Valores absolutos.

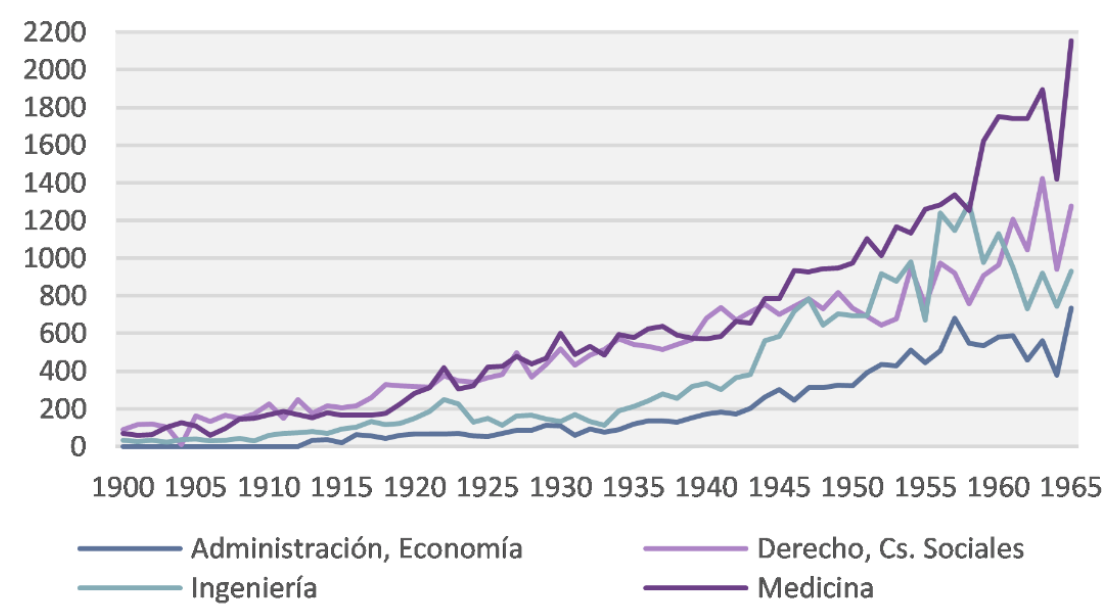

Fuente: Elaboración propia en base a Evolución de la mujer en las profesiones liberales en la Argentina, años 1900-1965, Oficina Nacional de la Mujer, 1970.

Aquel orden de la titulación masculina se mantuvo prácticamente durante todo el período. La excepción se dio entre 1950 y 1960, lapso en el que Ingeniería desplazó a Derecho al tercer puesto de los egresos masculinos. La Figura 4 ilustra el modo en que, justamente en esos años, la titulación femenina en Derecho crecía aceleradamente, y achicaba la brecha entre ambas curvas. 


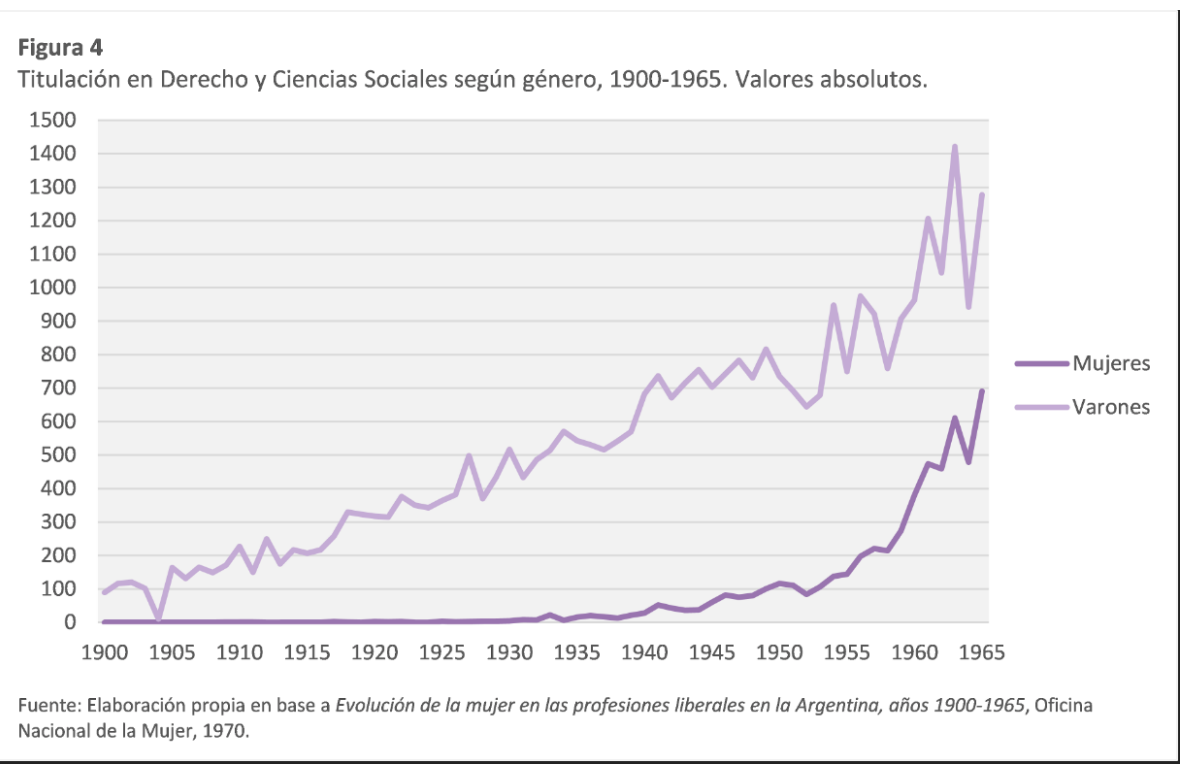

\section{Reorientación de las preferencias femeninas: la carrera de Derecho}

En Argentina, la primera mujer en obtener el título de abogada fue María Angélica Barreda. Egresada en 1909 de la Facultad de Ciencias Jurídicas y Sociales de la Universidad Nacional de La Plata. La inserción profesional no le resultó sencilla: aunque ya contaba con el diploma, le negaron la inscripción en la matrícula bonaerense, sin la cual no podía ejercer la profesión libre. Leiva (2012) retrata los pormenores del episodio, del cual se desprenden algunos elementos a tener en cuenta. Si bien finalmente la Suprema Corte de la Provincia de Buenos Aires permitió la matriculación de Barreda, la negativa inicial y los debates que suscitó nos permiten conocer algo del contexto e imaginar rasgos de su experiencia universitaria. Quienes se manifestaban en contra de que las mujeres ejercieran la abogacía estaban preocupados por el impacto que podría tener sobre las instituciones, y temían, especialmente, que suscitara una reconfiguración de la organización familiar: "[para las mujeres] una cosa es el título y otra distinta el ejercicio profesional" (Leiva, 2012). Es interesante señalar que una de las voces detractoras de la matriculación de la joven abogada correspondía a un docente de la Universidad Nacional de La Plata, institución que había expedido el diploma de Barreda.

Afortunadamente, no sólo aquellas voces se hicieron escuchar. Arias (2017, pp. 43-46) analiza la gran repercusión que tuvo el caso en los medios, la cual desbordó incluso al ámbito local, al aparecer en la revista Caras y Caretas. Según la autora, las posturas allí expresadas fueron en su mayoría favorables a Barreda y, en el caso del diario platense El Día, se siguió de cerca, y en varias publicaciones, la evolución del proceso judicial. Sin embargo, resulta llamativo que la amplia difusión del episodio, al menos a nivel local, no tuvo al parecer efectos promotores de la abogacía entre las mujeres que ingresaban a la Universidad Nacional de La Plata. Como veremos en el próximo apartado, los egresos femeninos en la Facultad de Ciencias Jurídicas y Sociales platense se mantuvieron prácticamente en cero en los años posteriores a la graduación de Barreda.

Sin embargo, como dijimos, hacia mediados de siglo esto comenzaba a cambiar. La publicación de la Oficina Nacional de la Mujer ofrece datos que nos permiten distinguir algunas características de los procesos por universidad. En las universidades de La Plata, del Litoral y de Córdoba, en las cuales en 1960 estudiaba el $40 \%$ del estudiantado de las universidades nacionales (Departamento de Estadística Educativa, 1967, p. 88), las carreras de Derecho fueron las que más títulos les otorgaron a las estudiantes mujeres entre 1956 y 1965. Como indica el cuadro de la Figura 5, el primer puesto se lo lleva la Universidad Nacional de Córdoba, donde el 33 \% de la titulación femenina entre 1961 y 1965 correspondió a la carrera de Derecho, seguida por la 
Universidad Nacional de La Plata, con un 25,56\%. En ambos casos los porcentajes superaban la participación de Derecho en la titulación de las mujeres a nivel nacional, que alcanzaba el 21,36 \%.

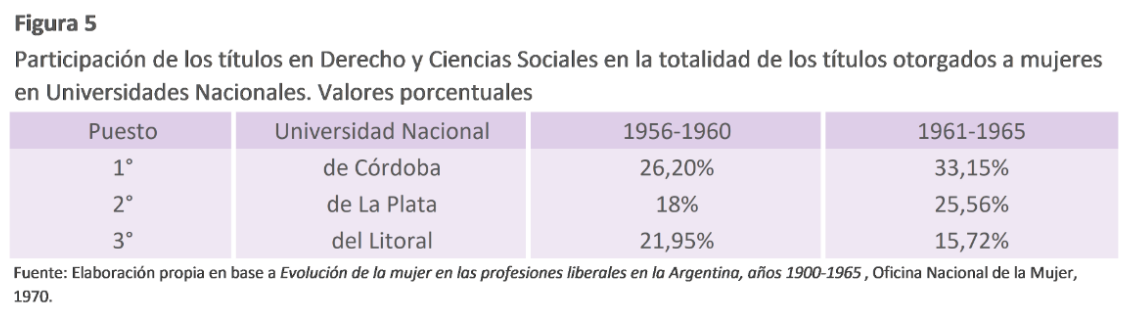

Respecto de la Universidad de Buenos Aires, que en 1960 representaba casi el $46 \%$ de la matrícula total de las universidades nacionales (Departamento de Estadística Educativa, 1967, p. 88), la carrera que contó con más egresos femeninos entre 1961 y 1965 fue Medicina, y Derecho quedó en un segundo puesto. Sin embargo, si miramos los números absolutos, fue la UBA la que más títulos en abogacía le otorgó a mujeres en aquel lapso: 1278 diplomas, contra 740 y 350 en Córdoba y La Plata, respectivamente.

Estos datos nos permiten constatar movimientos interesantes en relación a las mujeres y los estudios universitarios. No obstante, creemos que no alcanzan para realizar una caracterización integral de la participación femenina en la universidad.

\section{Limitaciones de la publicación}

Evolución de la mujer en las profesiones liberales en Argentina se ha posicionado como una fuente valiosa para el análisis de las universitarias en nuestro país. Ante la ausencia o escasez de datos sobre la matrícula por género, la evolución de la titulación detallada allí ha aportado a la comprensión del proceso. Sin embargo, creemos necesario reparar en las limitaciones que presenta.

El estudio estadístico se valió de los datos de titulación que las universidades nacionales remitieron. Los mismos se refieren a la cantidad de títulos expedidos y no a las personas egresadas. Esto presenta un primer inconveniente en su uso, ya que no sabemos con certeza cuántas fueron las mujeres que egresaron. Por otro lado, la Oficina Nacional de la Mujer decidió publicar las cantidades totales por año y por grupo de carrera; no sumó la variable universidad nacional, lo que nos limita su uso a quienes nos interesamos por una institución en concreto. Aquí es necesario subrayar, además, que el estudio deja afuera a las universidades privadas, vigentes en nuestro país desde 1958 (Buchbinder, 2010).

El hecho de no contar con la información en bruto que las universidades nacionales le enviaron a la Oficina trae aparejado un tercer obstáculo: debemos utilizar la clasificación por grupo de carreras elaborada por la dependencia. En el caso concreto de Derecho, por ejemplo, no distingue entre títulos de abogacía y escribanía, cuestión que nos interesaría poder dilucidar.

Además, aún si pudiéramos acceder a los datos en bruto, los mismos no bastarían para caracterizar la participación de las mujeres en la universidad. En primer lugar, porque nos seguiría faltando información sobre la matrícula discriminada por género, único modo de medir el ingreso femenino a la universidad. En segundo lugar, porque no podemos utilizarlos como indicadores de las preferencias de las mujeres: la titulación da cuenta de las carreras que más diplomas les otorgaron, no de las más elegidas por ellas. Por último, porque necesitamos acceder tanto a la titulación como a la matriculación para poder decir algo sobre la permanencia y la deserción de las mujeres en la universidad, así como también sobre la duración de los estudios.

Si bien estas dificultades limitan la comprensión del proceso de incorporación de las mujeres a las universidades a nivel nacional, existen alternativas que nos permiten cubrir algunos de estos vacíos estadísticos. Posar la mirada en una institución en concreto es una de ellas. De todos modos, esto no asegura 
el acceso sin obstáculos a la información con la que nos gustaría contar, puesto que el registro de la población estudiantil varía entre las universidades, e incluso entre las distintas unidades académicas en el interior de las mismas.

Las autoras que han tomado este camino desplegaron diferentes estrategias para acceder a la información cuantitativa que buscaban. Lorenzo (2016) puso el foco en la Universidad de Buenos Aires y caracterizó el paso de las universitarias a partir de datos relativos a los títulos, los exámenes de ingreso -y sus resultados-, y las matrículas de algunas facultades. Denot (2007) también reparó en la UBA, pero sólo en la Facultad de Filosofía y Letras. A partir de las tesis doctorales y las tesinas de grado reconstruyó la evolución de los egresos en esa facultad en las primeras décadas del siglo XX. García $(2011,2006)$ estudió a las universitarias de la Universidad Nacional de La Plata (UNLP), a partir del análisis de la participación femenina en algunas de sus unidades académicas, en particular en la Facultad de Ciencias Naturales y Museo, que cuenta con un Archivo Histórico muy importante, en el cual identificó datos sobre la matrícula, becas otorgadas a los y las estudiantes, así como también información relativa a las ayudantías en las cátedras. Arias (2017), interesada también en la UNLP, se valió de los listados de los actos de colación de la universidad para reconstruir la evolución de los egresos en las décadas de 1920 y 1930.

Vale aclarar que esta enumeración no pretende ser exhaustiva, sino citar ejemplos de los modos en que algunas autoras lograron caracterizar genéricamente a la población estudiantil en las instituciones en las que posaron la mirada. La misma intención la comparte el próximo apartado, en el cual presentaremos algunos avances del trabajo que realizamos a partir de información relativa a la titulación de la UNLP.

\section{LAS UNIVERSITARIAS DE LA UNLP}

\section{La Universidad Nacional de La Plata}

La Universidad de La Plata encuentra sus orígenes en estrecho vínculo con las expectativas que recaían sobre la nueva capital de la provincia de Buenos Aires. Vallejo (2007) relata los pormenores de esta relación y el modo en que La Plata se fue constituyendo en una "ciudad universitaria", mote con el que aun hoy la denominamos.

El plan fundacional de la ciudad se asentaba sobre proyectos orientados al desarrollo científico, que aspiraban -según el autor- a posicionarla por delante de la ciudad de Buenos Aires en el afán modernizador de la época. Sin embargo, estos programas encontraron tempranamente sus límites, en una crisis económica que se hizo sentir tanto como las internas entre el poder nacional y los representantes provinciales (Vallejo, 2007).

En este contexto dio sus primeros pasos la Universidad de La Plata bajo la órbita provincial. Inaugurada en 1897, contaba con cuatro unidades académicas: Derecho y Ciencias Sociales, Ciencias Médicas, Ciencias Físico-matemáticas, y Química y Farmacia. La Facultad de Derecho y Ciencias Sociales rápidamente pobló sus aulas: según narra el texto que publicó la UNLP en conmemoración de su centenario, grupos de vecinos que habían elevado a las autoridades el pedido por una universidad en La Plata, estaban particularmente interesados en la carrera de Derecho (Barba, 1998).

Esta cuestión trajo cola tiempo después cuando, en respuesta a la solicitud de dotar de validez nacional a los títulos expedidos por la Universidad Provincial, el entonces presidente Roca sentenció: "al gobierno no le interesan los abogados. Les aconsejo vayan a trabajar al campo" (citado por Vallejo, 2007, p. 190). Así pues, la orientación de los egresados no les incumbía sólo a ellos, desde sus propias búsquedas vocacionales y/o profesionales, sino que a su vez se erigía como una cuestión política que reafirmaba las rispideces entre la provincia y el gobierno nacional. Era además, como mencionamos en la introducción -siguiendo 
a Buchbinder (2010)-, un asunto que enfrentaba a las elites con las clases en ascenso, a las que mucho les interesaba el porvenir que un título de abogado podía ofrecerles.

Finalmente, en 1905, la universidad platense es nacionalizada. Vallejo (2007) destaca lo innovadora de la propuesta de Joaquín V. González -impulsor de su nacionalización-, que no incluyó en la estructura de la nueva Universidad a la Facultad de Ciencias Médicas. Buchbinder (2010) señala que el proyecto para la Facultad de Derecho y Ciencias Sociales, en sintonía con la decisión de omitir Medicina, "no asumiría solamente el papel de la formación de abogados sino que comprendería también los estudios científicos en materia social, moral y política". De este modo, el perfil de la UNLP se concebía en contraste con las orientaciones más "profesionalistas".

Estas decisiones por supuesto impactaron en la distribución de la población estudiantil platense, lo que la distinguió de aquello que ocurría a nivel nacional, donde se mantenía la preponderancia de Medicina que señalamos en el apartado previo. A continuación, expondremos con mayor detalle estas diferencias ya que presentaremos un avance del análisis que hemos realizado a partir de datos sobre la titulación de la UNLP, entre 1905 y 1972.

\section{Evolución de la titulación}

El estudio de una institución en particular puede ser, como dijimos, un camino útil a la hora de profundizar en las características de la población universitaria femenina si se sortean los vacíos de la información a nivel nacional. Sin embargo, esta posibilidad sólo existe si la universidad en cuestión conserva documentación al respecto y la ofrece para su consulta.

En el caso de la UNLP, las estadísticas publicadas sobre su población universitaria sólo abarcan los años de la última década. Es por ello que para llevar a cabo nuestra pesquisa nos valimos de documentación histórica. Afortunadamente, en septiembre de 2013 se creó el Archivo Histórico de la UNLP, que reúne, conserva y difunde los expedientes producidos por la Presidencia de la Universidad.

Allí nos facilitaron un registro de los títulos otorgados desde su nacionalización hasta 1972. El mismo fue realizado por la Dirección de Títulos y Planes, según indican las portadas de los tomos. Está presentado en forma de listas por año; cada listado corresponde a un año en particular y enumera los títulos que fueron otorgados en cantidades totales a varones y a mujeres. El formato se corresponde con el solicitado por la Oficina Nacional de la Mujer. Nos preguntamos si ese pedido habrá impulsado su confección.

Para poder analizar aquella información, primero hemos cargado los datos en una hoja de cálculo. Luego, comenzamos el proceso de normalización de las denominaciones, con el objetivo de lograr una base estructurada que nos permitiera comparar la información entre sí, a partir del cruce de diferentes variables. Para ello fue central el estudio particularizado de las unidades académicas, a fin de conocer la denominación de los títulos que otorgaban y cómo fueron cambiando -éstas y los títulos- a través de los años. En las líneas que siguen, expondremos algunos de nuestros avances en esta tarea que aun continuamos.

La Figura 6 ilustra la evolución de la titulación por género en el período analizado. Vemos allí que los egresos en la UNLP así como los totales nacionales tuvieron un crecimiento sostenido a lo largo de los años, con algunas depresiones aisladas que no modificaron sustancialmente la tendencia en ascenso. Si comparamos ambas curvas, observamos que la brecha comienza a cerrarse ya entrados los años cincuenta, como ocurrió a nivel nacional. Mientras que, en 1950, sólo el $16 \%$ de los títulos - 122 en valores absolutos- eran otorgados a mujeres, veinte años después, en 1970, los egresos femeninos representaron un tercio del total, hasta llegar a los 682 títulos. Para los varones, en cambio, la curva toma velocidad en los años posteriores a la Reforma Universitaria, cuando "La Plata se convirtió en un gran centro receptor de estudiantes del interior del país y de países limítrofes" (Vallejo, 2007, p. 331). 


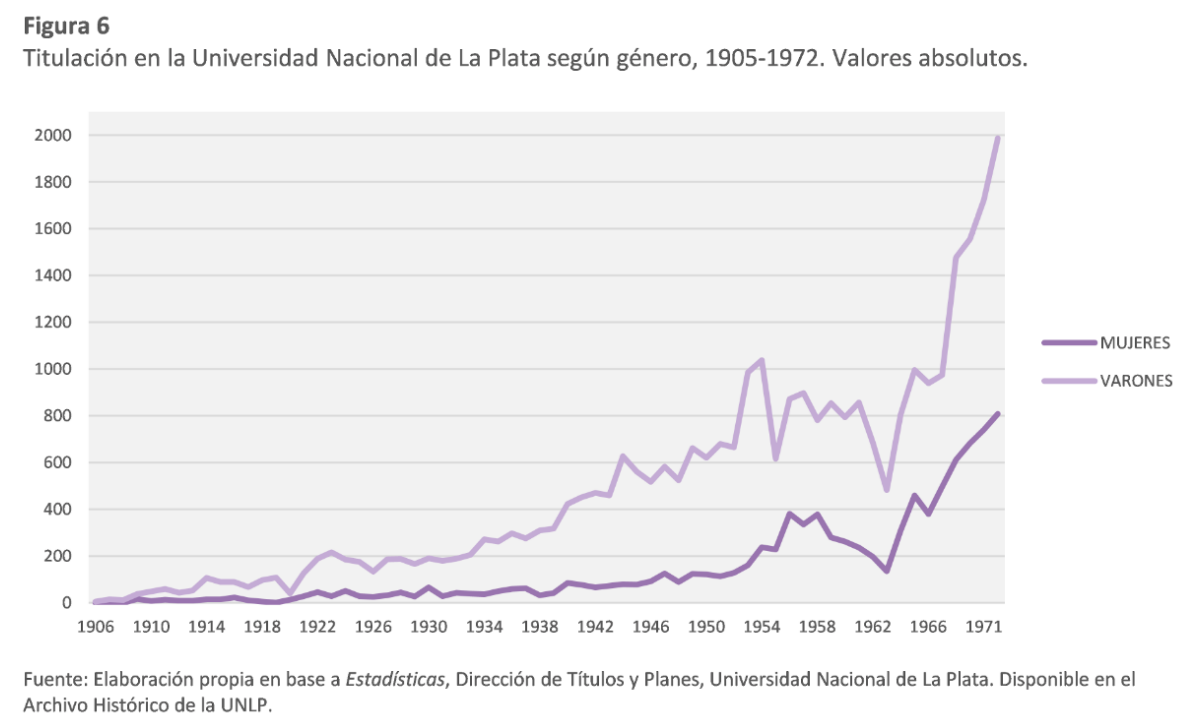

La presencia de mujeres en la Universidad de La Plata remonta a los años bajo la órbita provincial. García (2006) señala que la Facultad de Química y Farmacia admitía títulos de escuelas normales, lo que incentivaba a las mujeres a elegir aquella opción. Años más tarde, luego de la nacionalización de la Universidad, Farmacia se posicionó a la cabeza de los egresos femeninos, seguida por los profesorados, que también admitían estudiantes provenientes de escuelas normales.

En una primera etapa, los estudios de profesorado se nucleaban en la Sección Pedagógica que dependía de la Facultad de Ciencias Jurídicas y Sociales. Más tarde, en 1914, se creó la Facultad de Ciencias de la Educación, que en 1921 se convirtió en Humanidades y Ciencias de la Educación. La formación de los profesorados involucraba en muchos casos el cursar materias en otras facultades para obtener así la especialización que se deseaba enseñar (García, 2006). Distinto era el caso de la Escuela Superior de Bellas Artes que otorgaba sus propios títulos de profesorado.

Como observamos en la Figura 7, entre 1905 y 1930 la carrera con más egresos femeninos fue farmacia, que otorgó 231 títulos. La siguieron los profesorados que nucleaba la Facultad de Humanidades y Ciencias de la Educación, con 224 títulos. En tercer lugar aparecen los profesorados de Bellas Artes, con un total de 14 títulos, expedidos -vale aclarar- en sólo seis años ya que la Escuela fue creada en 1924. Más lejos se encuentra abogacía, con 9 egresadas. En comparación con los totales nacionales de la titulación femenina, la gran ausente es Medicina, que no se va a contar entre la oferta de la UNLP sino hasta 1934, cuando se crea la Facultad de Ciencias Médicas.

La Figura 8 representa los egresos de las mujeres en los años posteriores, entre 1931 y 1950. En este período, los profesorados le ganaron terreno a los títulos en Farmacia. A su vez, más egresos se distribuyeron entre otras carreras, tales como abogacía, con 69 títulos, y medicina, con 31. Acá también la diferencia con los parámetros nacionales se relaciona con la carrera de medicina, que osciló entre el primero y el segundo puesto a nivel nacional (Figura 2). 
Anuario del Instituto de Historia Argentina, junio 2018, vol. 18, n 1, e064. ISSN 2314-257X

Figura 7

Títulos otorgados a mujeres en la UNLP, 1905-1930.

valores absolutos

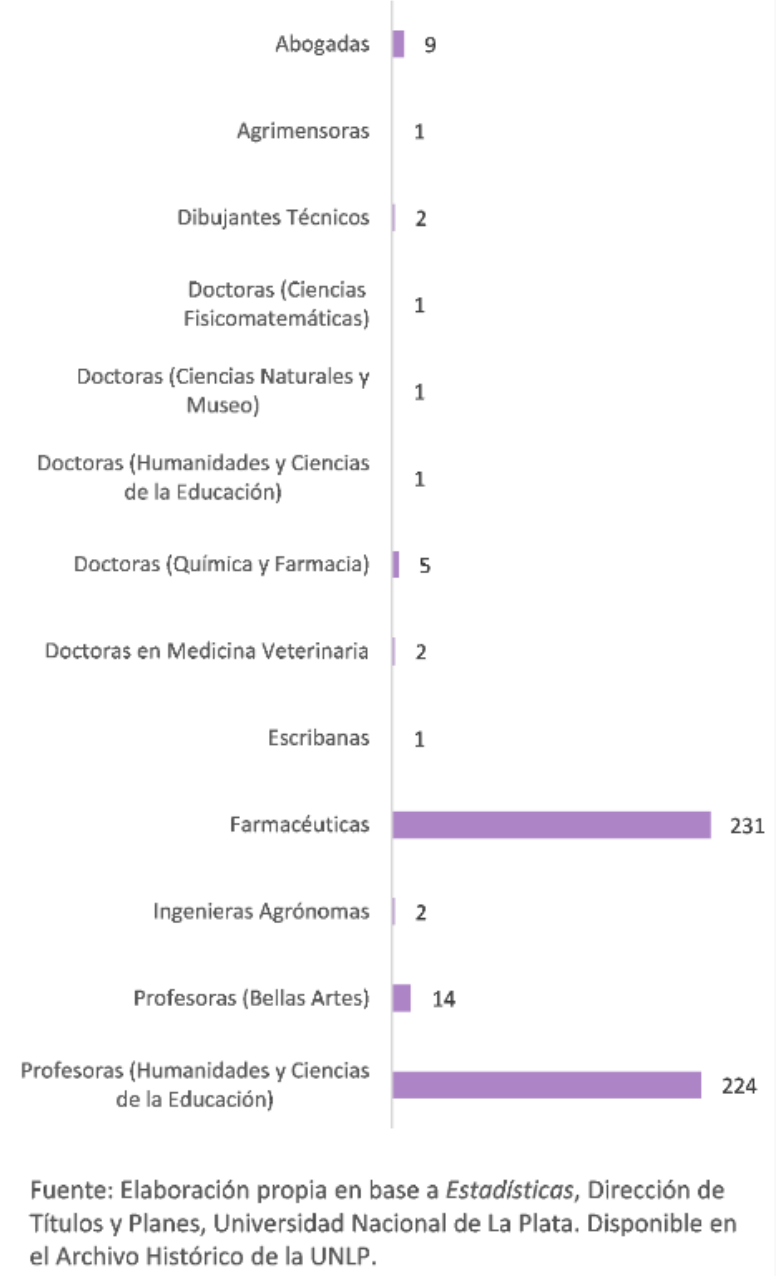




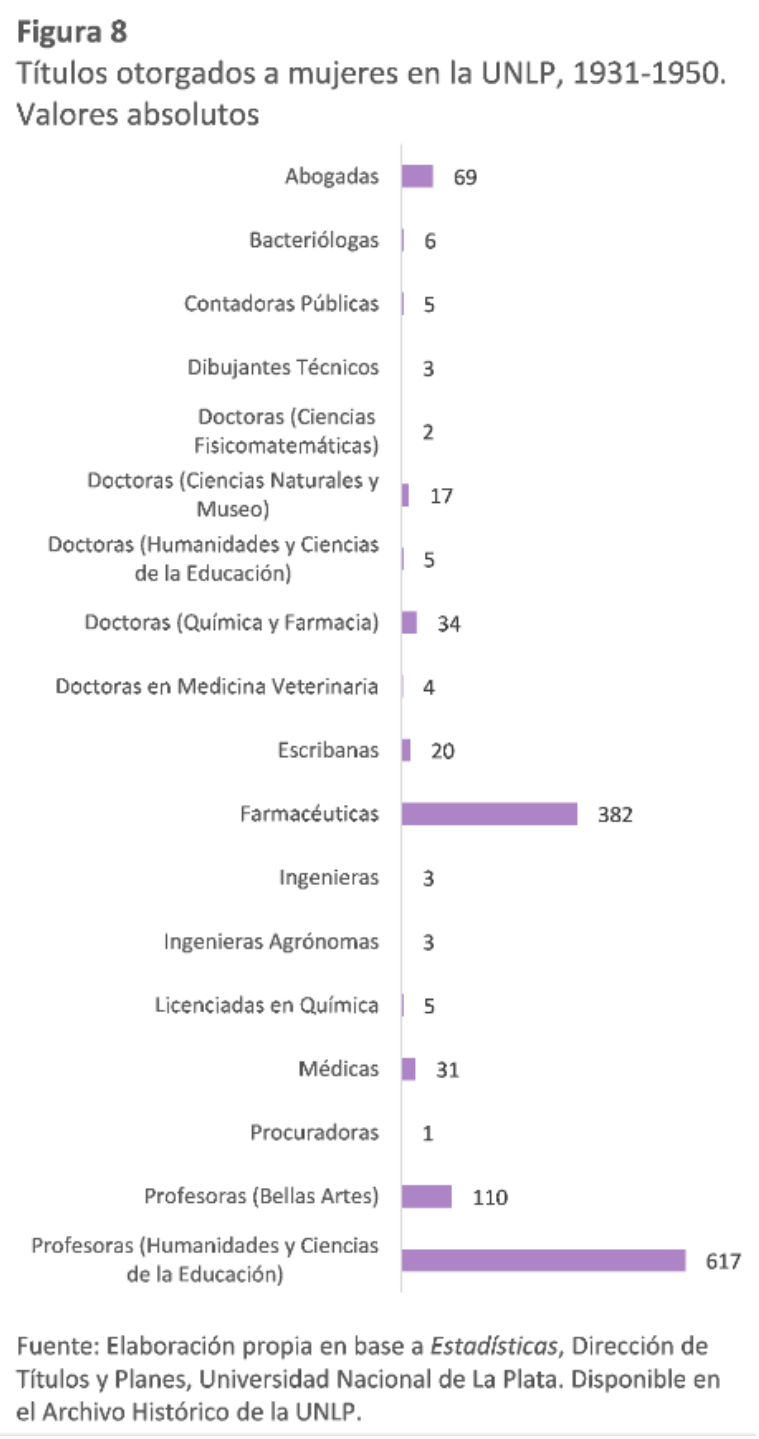

Los egresos del último período que comprende nuestro trabajo, 1951-1972, están ordenados en las Figura 9 y 10 según la unidad académica en lugar de según el título expedido. Esta decisión responde a fines meramente ilustrativos, ya que el gran incremento en la oferta de carreras que ocurrió por aquellos años dificultaba la presentación de los datos para los objetivos que aquí nos proponemos.

Observamos en la Figura 9 el aumento notable de la titulación femenina en las facultades de Ciencias Médicas y de Ciencias Jurídicas y Sociales, con 1887 y 1414 títulos respectivamente. La reorientación de los egresos de las mujeres que señalamos a nivel nacional también tuvo lugar en la UNLP. La Facultad de Humanidades y Ciencias de la Educación se mantuvo en el podio. Con una gran oferta de licenciaturas, sumada a los profesorados y la incorporación de la carrera de Psicología expidió 1694 títulos en el período. La Facultad de Ciencias Exactas, donde se cursaba la carrera de Farmacia, también se posicionó entre las facultades que más títulos les otorgaron a las universitarias platenses. 
Figura 9

Títulos otorgados a mujeres en la UNLP, según Facultad, 1951-1972. Valores absolutos

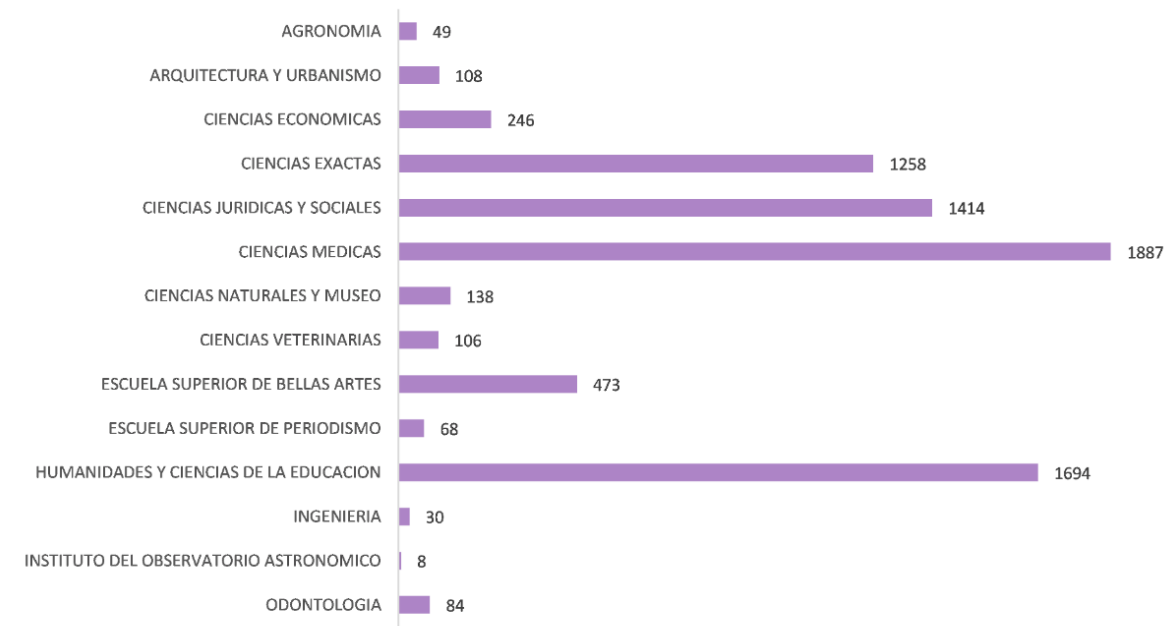

Fuente: Elaboración propia en base a Estadísticas, Dirección de Títulos y Planes, Universidad Nacional de La Plata. Disponible en el Archivo Histórico de la UNLP.

Figura 10

Títulos otorgados a varones en la UNLP, según Facultad, 1951-1972. Valores absolutos

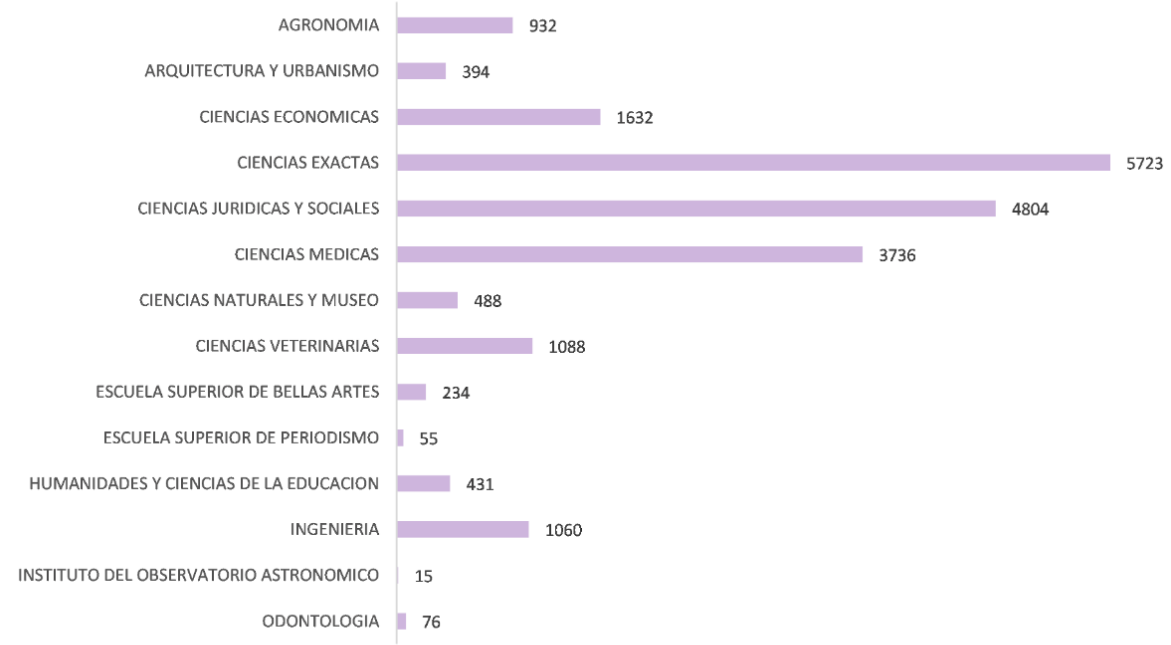

Fuente: Elaboración propia en base a Estadísticas, Dirección de Títulos y Planes, Universidad Nacional de La Plata. Disponible en el Archivo Histórico de la UNLP.

La Figura 10 representa los egresos de los varones en el mismo período. Vemos allí que la Facultad de Ciencias Exactas fue la que más títulos les otorgó, seguida por la de Ciencias Jurídicas y Sociales, y luego por la de Ciencias Médicas. En este caso la diferencia con los totales nacionales es notable, ya que éstos indicaban una preponderancia marcada de Medicina, seguida por Derecho e Ingeniería. Por el contrario, ésta última, en el caso platense se posicionó incluso por debajo de las carreras de Ciencias Económicas.

Presentado el panorama general, a continuación examinaremos con mayor detalle los títulos otorgados por la Facultad de Ciencias Jurídicas y Sociales. 


\section{La Facultad de Ciencias Jurídicas y Sociales}

Como ya mencionamos, la primera abogada argentina egresó de esta Facultad en 1909. Por aquellos años eran muy pocas las mujeres que frecuentaban sus aulas, sin embargo su presencia causaba cierto revuelo. En la Memoria de 1906, el decano de la facultad Rodolfo Rivarola se ocupa de un "tema que me ha sido sugerido por las advertencias y observaciones de algunos profesores" (Rivarola, 1906, p. 81): la presencia de tres estudiantes mujeres en la facultad. Rivarola relata la decepción de algunos docentes respecto del desempeño de las alumnas, ya que "no satisfacían un anhelo de superioridad respecto de la medianía de aquellos [sus compañeros varones], que los profesores habrían deseado como justificación de un hecho nuevo en nuestra historia universitaria" (Rivarola, 1906, p. 89). La postura del decano no avala estos planteos, sino que es favorable a la incorporación de mujeres a los estudios de derecho e inclusive las proyecta profesionalmente. Esto demuestra que había posiciones encontradas en el interior de la facultad.

De todos modos, la titulación de mujeres se mantuvo en niveles muy bajos durante un período prolongado. Fue recién en la década de los cuarenta cuando comenzó a repuntar, para consolidar su tendencia en ascenso en los años cincuenta (Figura 11). Si entre 1905 y 1950 habían egresado sólo 138 mujeres, en la década de los cincuenta esa cifra se había triplicado, y mantuvo el ritmo en los años sesenta.

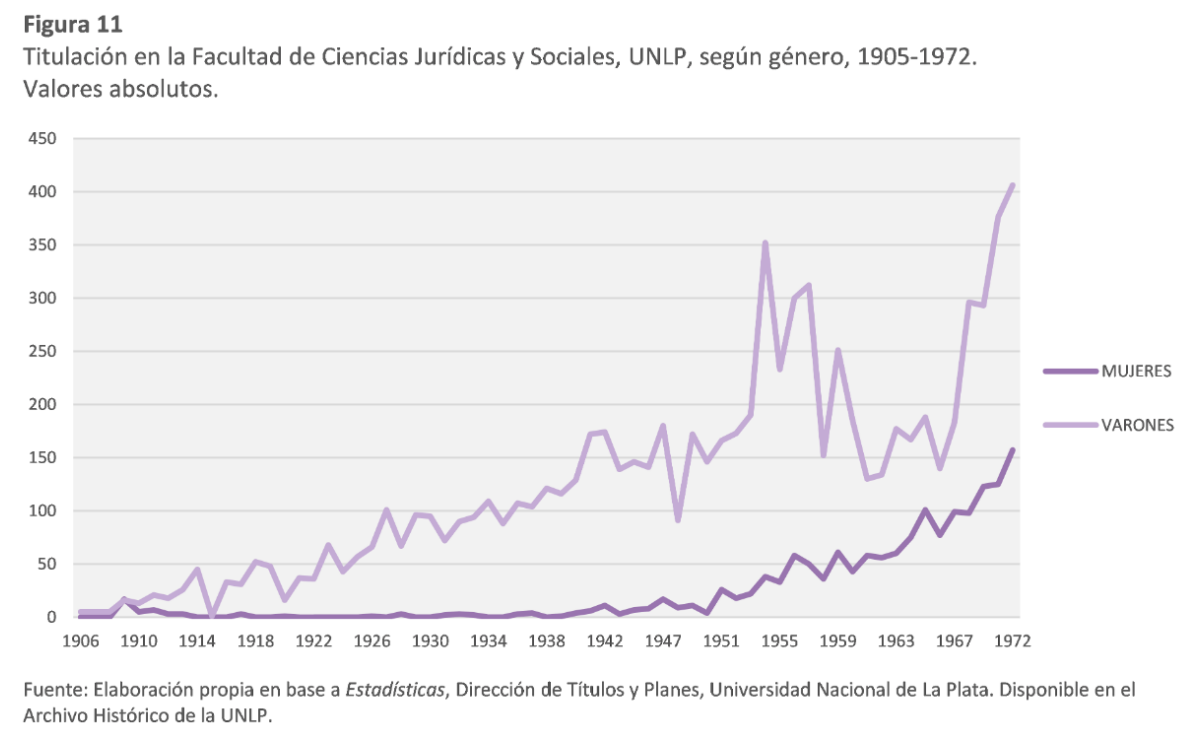

Un rasgo interesante del proceso se evidencia cuando examinamos los títulos en detalle. Como observamos en la Figura 12, el incremento en la titulación femenina de los años sesenta fue protagonizado por la carrera de Escribanía y no por la de Derecho, a diferencia de la preponderancia de esta última en los egresos masculinos, ilustrados en la Figura 13. 

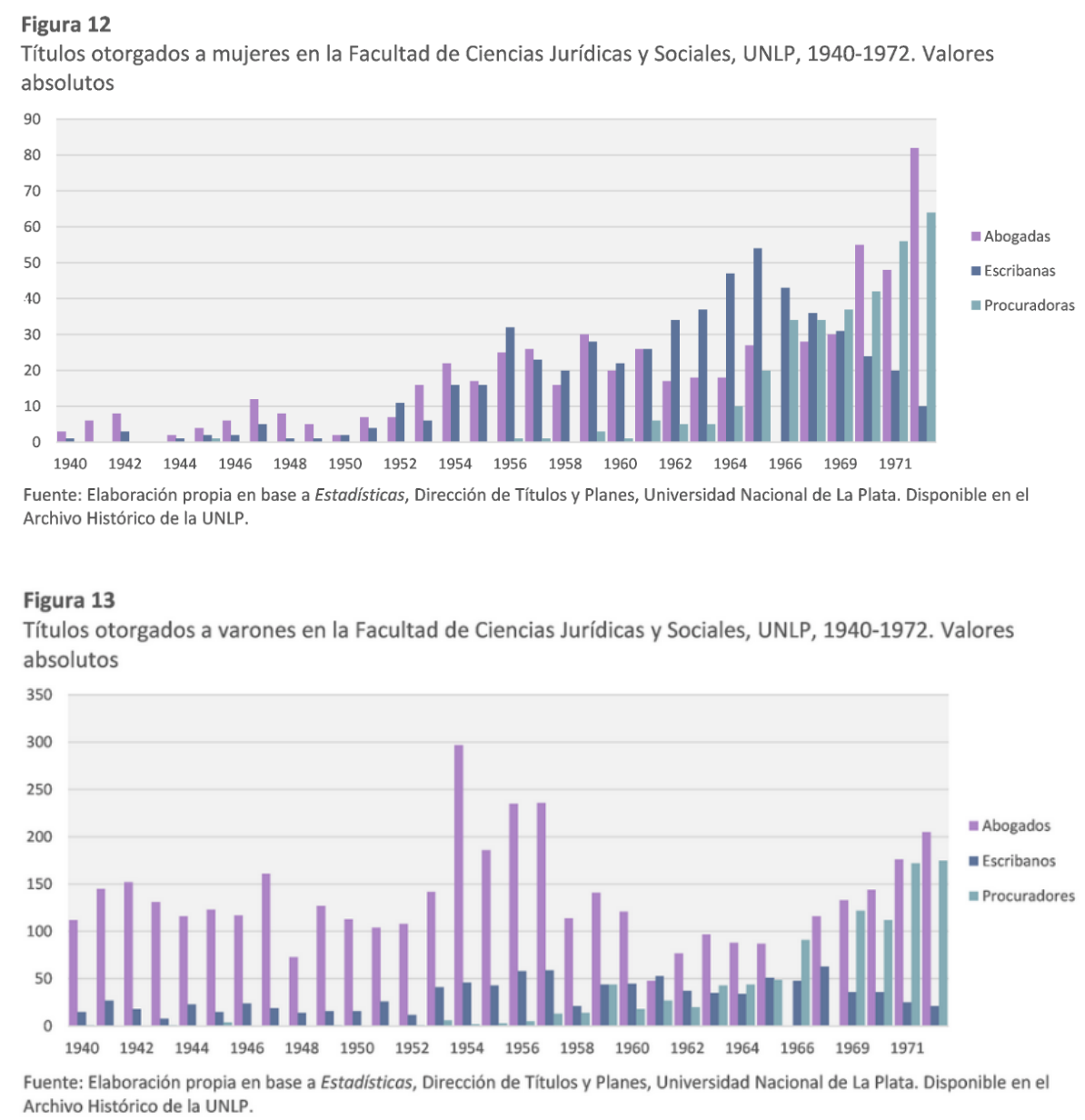

Esta constatación nos despertó la pregunta sobre los motivos por los cuales las mujeres se abocaron hacia la carrera de Escribanía. Una respuesta posible puede estar relacionada con la duración de los estudios: en aquella época, las materias requeridas para obtener el título de escribano/a eran menos que las solicitadas para derecho. De todos modos, no creemos que ésta fuera la única razón; por ello continuamos estudiando esta cuestión, reparando en el modo en que los perfiles profesionales de las carreras jurídicas se fueron conformando.

\section{ReFLEXiONES FINALES}

Las cuestiones aquí planteadas intentan ilustrar nuestro derrotero en el análisis de una dimensión del fenómeno de incorporación de las mujeres a la universidad: la cuantitativa. Se trata de un recorte que de ningún modo ofrece una comprensión acabada del proceso, sino que -a nuestro modo de ver- permite contextualizar su abordaje y, en algunos casos como en el nuestro, también oficia de disparador de una pregunta de investigación. Si bien nuestro trabajo no se inscribe en los estudios sobre universidad, esta temática nos alcanzó a la hora de querer caracterizar el paso de las profesionales del derecho por los estudios universitarios y, además, nos ayudó a delimitar nuestro período de análisis al constatar el notable incremento de mujeres en las carreras de derecho hacia fines de los años cincuenta.

En este escrito intentamos exponer los desafíos que se presentan para quienes nos proponemos estudiar la incorporación de las mujeres a los estudios universitarios en Argentina. Los obstáculos con los que nos encontramos nos llevaron a indagar con mayor profundidad en las fuentes estadísticas disponibles y a decidir circunscribir nuestro estudio a una institución en particular: la Universidad Nacional de La Plata. 
En primer lugar, revisamos las fuentes estadísticas que analizan la población universitaria a nivel nacional. Encontramos allí una ausencia remarcable de datos discriminados por género, como ya otras autoras habían señalado. La excepción la constituye Evolución de la mujer en las profesiones liberales en Argentina, 1900-1965, estudio publicado por la Oficina Nacional de la Mujer en los años sesenta. Expusimos el modo en que la información allí volcada ofrece la posibilidad de examinar los movimientos en los egresos femeninos de las universidades nacionales en una serie de largo alcance.

Luego, reparamos en las limitaciones que hallamos, algunas que hemos podido sortear y otras aún pendientes, como, por ejemplo, la caracterización de la matrícula universitaria femenina a lo largo del siglo XX. En el recorrido por el que estas indagaciones nos llevaron pudimos comprender las dificultades particulares de este objetivo, que presenta desafíos incluso para su abordaje global, es decir, sin la distinción de género. La falta de información sobre la matrícula y -podemos agregar-sobre los ingresos obtura el análisis cuantitativo de otras dimensiones de la participación universitaria de las mujeres, como son la permanencia y la duración de sus estudios.

Finalmente, presentamos el relevamiento que realizamos sobre los egresos de la UNLP con la intención de establecer una comparación con los totales nacionales, así como también con el objetivo de sortear algunos de los límites que encontrábamos en la información estadística disponible. Esta tarea nos permitió resolver inconvenientes tales como la ausencia de datos de titulación en valores absolutos desagregados por universidad nacional, y la clasificación por grupo de carreras, que impedía la distinción de orientaciones dentro de cada grupo. Así, pudimos identificar una cuestión en la que profundizaremos: la escribanía como opción predilecta de las profesionales del derecho en nuestro período de análisis.

Entonces, este estudio busca exponer un problema metodológico vinculado a las fuentes estadísticas universitarias y los desafíos para hallar allí a las mujeres. Asimismo, introduce nuestros avances en la identificación y análisis de las fuentes de la UNLP. Esta tarea implicó transformar registros administrativos de la Universidad en información estadística que da cuenta de la evolución de los egresos de mujeres y varones, y de las orientaciones profesionales de los mismos, entre 1905 y 1972 . Creemos que este avance, aunque inicial y a la espera de una mayor dedicación, propicia el surgimiento de nuevos interrogantes en relación a la población universitaria de la UNLP, y ofrece, a su vez, algunas respuestas.

\section{Agradecimientos}

Quiero agradecer a Laura Casareto, Myriam Hara y las demás integrantes del Archivo Histórico de la Universidad Nacional de La Plata por su ayuda predispuesta y generosa. También mencionar que gran parte de este trabajo fue realizado gracias a la beca que me otorgó la Comisión de Investigaciones Científicas de la Provincia de Buenos Aires para el año 2017.

\section{FUENTES}

Estadísticas, 1905-1972, Dirección de Títulos y Planes, Universidad Nacional de La Plata. Disponible en el Archivo Histórico de la UNLP, http://archivohistorico.unlp.edu.ar/

Expediente nº 1537 (1962), Universidad Nacional de La Plata. Disponible en el Archivo Histórico de la UNLP, htt p://archivohistorico.unlp.edu.ar/

\section{BiBLiografía}

Aráoz, A. (1968). Comentarios sobre el trabajo del Dr. Julio Olivera: La Universidad como unidad de producción. Revista Económica, 2(1), 111-114. 
Arias, A. C. (2017). Mujeres universitarias en la Argentina. Algunas cuestiones acerca de la Universidad Nacional de La Plata en las primeras décadas de siglo XX. Trabajo final de la Especialización en Educación en Géneros y Sexualidades de la UNLP.

Barba, E. F. (1998). La Universidad Nacional de La Plata en su centenario 1897-1997. La Plata: Universidad Nacional de La Plata

Barrancos, D. (2008). Mujeres, entre la casa y la plaza. Buenos Aires: Sudamericana.

Barrancos, D. (2012). Mujeres en la sociedad argentina: una historia de cinco siglos. Buenos Aires: Sudamericana.

Buchbinder, P. (2010). Historia de las universidades argentina. Buenos Aires: Sudamericana.

Cammarota, A. (2014). Somos Bachiyeres. Juventud, cultura escolar y peronismo en el Colegio Nacional Mixto de Morón (1949-1969). Ciudad Autónoma de Buenos Aires: Biblos.

Cano, D. J. (1985). La educación superior en Argentina. Buenos Aires: Flacso - CRESALC/Unesco.

Chiroleu, A. (2006). Las paradojas de la modernización universitaria de los años 60. Estudios Sociales. Revista Universitaria Semestral. Universidad Nacional del Litoral, XVI (30), 97-126.

CONADE (1968). Educación, recursos humanos y desarrollo económico-social. Buenos Aires: Secretaría del Consejo Nacional de Desarrollo. Presidencia de la Nación.

Cosse, I. (2010). Pareja, sexualidad y familia en los años sesenta. Una revolución discreta en Buenos Aires. Buenos Aires: Siglo Veintiuno Editores.

Daniel, C. (2009). El estado argentino y sus estadísticas. El derrotero de un largo proceso de institucionalización (18641968). Illapa, Revista Latinoamericana de Ciencias Sociales, 2, 151-174.

Denot, S. (2007). La emergencia de las mujeres en la Universidad de Buenos Aires: transformaciones en el campo intelectual y nuevos sujetos, 1889-1930. En V Encuentro Nacional y II Latinoamericano La Universidad como objeto de investigación. Tandil: Universidad Nacional del Centro de la Provincia de Buenos Aires.

Departamento de Estadística Educativa. (1967). Argentina. La educación en cifras, 1958-1967. Buenos Aires.

García Frinchaboy, M. (1981). Evolución de la participación universitaria femenina en Argentina (1940-1980). Buenos Aires: Departamento de Sociología. Universidad Católica Argentina.

García, S. V. (2011). Mujeres, ciencias naturales y empleo académico en la Argentina (1900-1940). R. Inter. Interdisc. INTERthesis, 8(2), 83-103. https://doi.org/10.50071807-1384.2011v8n2p83

García, S. V. (2006). Ni solas ni resignadas: la participación femenina en las actividades científico-académicas de la Argentina en los inicios del siglo XX. Cadernospagu, (27), 133-172. Recuperado a partir de http:// www.scielo.br/pdf/cpa/n27/32141.pdf

Germani, G., y Sautu, R. (1965). Regularidad y origen social en los estudiantes universitarios. Buenos Aires: Universidad de Buenos Aires.

Graciarena, J. (1961).Diseño de un modelo de investigación sobre la deserción estudiantil universitaria (Textos y d). Santa Fe: Departamento de Pedagogía Universitaria. Universidad Nacional del Litoral.

Leiva, A. D. (2012). La matriculación de la primera abogada argentina\#: María Angélica Barreda. Prudentia Iuris, (74), 201-213. Recuperado a partir de http://bibliotecadigital.uca.edu.ar/repositorio/revistas/matriculacion-primer a-abogada-argentina-barreda.pdf

Lionetti, L. (2005). La función republicana de la escuela pública: la formación del ciudadano en argentina a fines del siglo XIX. Revista mexicana de Investigación Educativa, X(27), 1-32. Recuperado a partir de https://biblat.unam.mx/es/revista/revista-mexicana-de-investigacion-educativa/articulo/la-funcion-republi cana-de-la-escuela-publica-la-formacion-del-ciudadano-en-argentina-a-fines-del-siglo-xix

Lorenzo, M. F. (2016). Que sepa coser, que sepa bordar, que sepa abrir la puerta para ir a la Universidad: las académicas de la Universidad de Buenos Aires en la primera mitad del siglo XX. Ciudad Autónoma de Buenos Aires: Eudeba.

Maglie, G., y García Frinchaboy, M. (1988). Situación educativa de las mujeres en Argentina. Buenos Aires: Subsecretaría de la Mujer de la Nación; UNICEF.

Nari, M. (1995). La educación de la mujer (o acerca de cómo cocinar y cambiar los pañales a su bebé de manera científica). Revista Mora, (1), 31-45. 
Novick, S. (1993). Mujer, Estado y políticas sociales. Buenos Aires: Centro Editor de América Latina S.A.

Oficina Nacional de la Mujer (1970). Evolución de la mujer en las profesiones liberales en Argentina. Buenos Aires: Oficina Nacional de la Mujer. Secretaria de Estado de Trabajo.

Palermo, A. I. (2006). El acceso de las mujeres a la educación universitaria. Revista Argentina de Sociología, 4(7), 11-46.

Perez Lindo, A. (1985). Universidad, politica y sociedad. Buenos Aires: EUDEBA S. E. M.

Queirolo, G. (2008). El mundo de las empleadas administrativas: perfiles laborales y carreras individuales (Buenos Aires, 1920-1940). Trabajos y Comunicaciones (2a Época), 8(34), 129-151. Recuperado a partir de http://www .fuentesmemoria.fahce.unlp.edu.ar/art_revistas/pr.3727/pr.3727.pdf

Queirolo, G. (2010). "Malos pasos" y "promociones”. Aproximaciones al trabajo femenino asalariado desde la historia y la literatura (Buenos Aires, 1919-1939). Anuario de la Escuela de Historia de la Facultad de Humanidades y Artes - Universidad Nacional de Rosario, (22), 53-80.

Ramacciotti, K., y Valobra, A. (2011). Modernas esculapios: acción política e inserción profesional, 1900-1950. Asociación de Historiadores Latinoamericanistas Europeos Publicación, (7), 23-51.

Rivarola, R. (1906). Facultad de Ciencias Jurídicas y Sociales en el año 1906. Memoria redactada por el Decano. La Plata: Universidad Nacional de La Plata.

Santos Sharpe, A. (2016). Un análisis histórico del abordaje sobre el abandono universitario en Argentina. Anuario de Historia de la Educación, 17(2), 3-31. Recuperado a partir de http://www.scielo.org.ar/pdf/histed/v17n2/v $17 \mathrm{n} 2 \mathrm{a} 02 . \mathrm{pdf}$

Suasnábar, C. (2014). Tradición e internacionalidad\#: Archivos de Ciencias de la Educación (3ra. Época) y el pensamiento educacional en los años sesenta. Archivos de Ciencias de la Educación, 8 (8), 1-23. Recuperado a partir de http://www.archivosdeciencias.fahce.unlp.edu.ar/article/view/Archivos08a05

Vallejo, G. (2007). Escenarios de la cultura cientifica argentina. Ciudad y universidad (1882-1955). Madrid: Consejo Superior de Investigaciones Científicas. 